\title{
Vivre en ligne en Algérie
}

Living online in Algéria

\section{Patrice Flichy}

\section{OpenEdition}

\section{Journals}

Édition électronique

URL : http://journals.openedition.org/ctd/1968

DOI : $10.4000 /$ ctd. 1968

ISSN : 2491-1437

\section{Éditeur}

Chaire Unesco Pratiques émergentes en technologies et communication pour le développement

\section{Édition imprimée}

Date de publication : 12 novembre 2015

\section{Référence électronique}

Patrice Flichy, «Vivre en ligne en Algérie », Communication, technologies et développement [En ligne], 2 |

2015, mis en ligne le 11 juin 2019, consulté le 22 juillet 2020. URL : http://journals.openedition.org/ ctd/1968; DOI : https://doi.org/10.4000/ctd.1968

Ce document a été généré automatiquement le 22 juillet 2020.

Communication, technologies et développement 


\title{
Vivre en ligne en Algérie
}

\author{
Living online in Algéria
}

\section{Patrice Flichy}

1 Comment s'organise la vie en ligne en Afrique ? Je voudrais apporter une contribution à cette réflexion, en présentant une monographie réalisée auprès de jeunes algériens qui

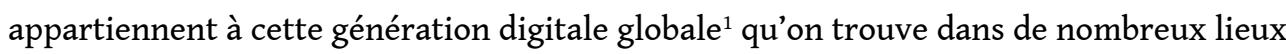
de notre planète. Il s'agit ici de jeunes algériens francophones, étudiants et lycéens à Alger, Constantine et Annaba ${ }^{2}$. La plupart d'entre eux sont issus des classes moyennes intellectuelles, ce sont des étudiants brillants qui consacrent beaucoup de temps à leurs études. Les lycéens appartiennent à un établissement d'excellence, Aristote et ressemblent beaucoup à ceux du lycée Boileau (Paris) étudié par Dominique Pasquier ${ }^{3}$, ils vivent dans un entre soi très particulier qui permet de s'évader de la culture vécue par bien des jeunes de leur âge en Algérie. Ils ont un goût pour la culture littéraire (lecture de la presse de qualité, de la littérature) un attrait pour les séries américaines, des goûts musicaux éclectiques. La plupart des lycéens comme des étudiants aimeraient continuer leurs études à l'étranger et notamment en France. Ils possèdent tous les outils nécessaires à la culture numérique ${ }^{4}$. Je présente, également, en contrepoint un groupe de jeunes chômeurs issus des classes populaires.

2 Cette monographique est intéressante à trois titres :

- Le rapport entre l'intérieur et l'extérieur constitue un cas limite, puisque l'extérieur a été inaccessible, en Algérie, pendant les années 1990, celle du terrorisme de la décennie noire. Aujourd'hui, le monde extérieur reste difficilement accessible aux filles.

- L'espace public (dans les deux sens du terme) est restreint. Il y a très peu d'équipements culturels publics (bibliothèques, cinémas...). L'espace du débat politique est réduit pour l'essentiel à la presse. L'activité des partis politiques d'opposition est très limitée.

- Ces jeunes se sentent enfermés dans le monde culturel algérien. Ils aspirent à aller dans un monde extérieur qu'ils parcourent, en ligne, dans tous les sens.

3 Les pratiques de ces jeunes sont placées dans un espace privé individuel, c'est le premier point que j'étudierai, elles sont au centre d'une tension permanente entre l'intérieur et l'extérieur (point 2). Ces jeunes trouvent également dans le numérique 
des ressources nouvelles pour mener leurs études, leurs loisirs (point 3) et leurs passions (point 4).

\section{L'individuel, le privé et le public}

4 Depuis trois quarts de siècle, les différents outils de communication ont suivi la même évolution. Ils ont, tout d'abord, remplacé les loisirs réalisés à l'extérieur, dans des lieux publics par des activités se déroulant dans l'espace domestique. La radio et le disque ont pris la place de la musique dans la rue ou dans des lieux publics (salles, cafés...), la télévision celle du cinéma. Si en Europe, cette évolution est complexe, puisque la télévision n'a pas complètement tué le cinéma. En France, après une forte baisse, au début de la télévision, sa fréquentation a ensuite stagné. Les concerts rencontrent également un vrai succès auprès des jeunes et leur public a augmenté ces dernières années ${ }^{5}$. En Algérie, il semble qu'au contraire, le domicile se soit substitué complètement à la salle de spectacle, d'abord pour des raisons économiques, la télévision et la radio sont de loin les loisirs les moins chers, mais aussi pour des raisons spécifiques, la peur et l'inquiétude liées au terrorisme de la «décennie noire " et l'encadrement plus fort qu'ailleurs des sorties des filles.

Le domicile a constitué un entre soi protecteur pour cette jeunesse algérienne qui a vécu son enfance dans une situation particulièrement difficile, les années 1990. Les attentats permanents, le couvre-feu obligeaient les jeunes à rester chez eux. «Moi, je n'ai pas joué dans la rue. Je n'ai pas vu ça. On avait peur quand on allait à l'école, on avait peur quand on allait à la plage. C'était à chaque fois des tueries, des bombes, des égorgements " se souvient Amine ${ }^{6}$, (25 ans Alger). De même un interne en médecine de Constantine qui était enfant avant la décennie noire se souvient qu'alors « on sortait avec la famille, il y avait une très belle forêt, le Djebel Ouahch, avec des lacs et tout ça, maintenant si vous vous aventurez, vous risquez l'agression à coup sûr » (Kamel, 32 ans).

6 L'injonction à rester chez soi a perduré dans les années 2000, d'autant plus que les sorties et lieux publics proposés aux jeunes sont souvent fort limités. Mais, cette injonction est beaucoup plus forte pour les filles que pour les garçons. Néanmoins, certains garçons vont à l'extérieur. «Je sors beaucoup. Je vais à des concerts, je fais beaucoup de sport » déclare Omar, étudiant à Constantine. Ahmed élève de terminal à Alger indique, quant à lui : « quand je reste chez moi, c'est pour me connecter ou bien rester un peu avec ma famille ou pour dormir. Ce n'est pas plus... sinon je sors dehors, je m'éclate avec mes amis ». De même les chômeurs d'Alger vont assister à des matchs et pratiquent le sport.

7 La situation est fort différente pour les filles. «Ici, à Constantine, pour des raisons de sécurité, une femme ne peut pas sortir à plus de 18 heures » estime Amina qui précise également qu'elle ne peut pas aller dans un bar seule. "Je prends chaque jour un café avec mon ami, s'il ne vient pas, je ne prends pas de café». On voit ainsi que dans l'espace public, il peut y avoir une violence faite aux femmes par les regards. A Alger, c'est à première vue différent, « il y a des filles qui s'assoient sur les terrasses » constate Amina. De même, les élèves du lycée Aristote sortent les unes chez les autres, vont en groupe sur la plage. Néanmoins, une jeune algéroise (Zina, 19 ans) indique que «cela n'est pas évident de sortir ». Aicha a intériorisé ce comportement féminin et déclare : « je suis plus à l'aise à la maison, je préfère la maison ». Quant à Yasmina, (20 
ans), elle a décidé de s'adapter à ce contrôle familial : «On est contraint de rester à la maison; donc on se trouve des loisirs ». Cette permanence des filles au domicile ne les empêche pas, comme nous le verrons, de communiquer avec l'extérieur. Nous retrouvons un comportement déjà observé avec le téléphone chez des adolescentes parisiennes. Une enquête de 2000 constate que comme les adolescentes pouvaient sortir deux fois moins le soir que les garçons, elles téléphonaient beaucoup plus à leurs amies $^{7}$.

8 Le phénomène de privatisation des loisirs qui caractérise l'époque contemporaine est encore plus fort en Algérie qu'ailleurs. Un deuxième phénomène s'ajoute à cette pratique privée des loisirs, leur individualisation ${ }^{8}$. A chacun, son appareil de communication. Cette évolution est d'abord apparue, en Europe, avec la radio, dans les années 1950-1960, puis plus lentement avec la télévision, le grand téléviseur du living room gardant longtemps un statut plus collectif. Il en a été ensuite de même avec le micro-ordinateur et le téléphone. Cette pratique individuelle des outils électroniques est, ainsi, la norme chez la plupart de nos enquêtés. Les micro-ordinateurs portables sont devenus pour ces jeunes un instrument individuel presque toujours connecté à internet qui de plus en plus remplace la télévision pour regarder les films et les séries.

9 La pratique d'internet chez ces jeunes algériens est une bonne illustration de cette évolution des pratiques de communication. Elle est passée en une dizaine d'années de l'espace public à l'espace individuel. Une évolution en accéléré en quelque sorte qu'on peut décrire ainsi : Au début des années 2000 , alors que la décennie noire s'achève, les cybercafés commencent à fleurir. " $\mathrm{Y}$ avait plein de cyber à Alger. Tout le monde se connectait ... On essayait de vivre un monde virtuel, un monde bien, un monde calme, sans guerre » (Amine). «L'apport d'internet est énorme, parce qu'à l'époque aussi, on n'avait pas la possibilité de sortir. C'était une fenêtre sur le monde » (Bachir, étudiant Alger). Mais petit à petit, la connexion domestique devient la situation standard ${ }^{9}$. Puis à la fin des années 2000 , ces jeunes se sont équipés de leur propre machine. Ils possèdent leur ordinateur personnel connecté à internet. Leurs activités d'internautes sont très individualisées. Ceux qui partagent un ordinateur collectif négocient une plage de temps avec leurs proches, les autres gèrent donc leur appareil comme il le souhaite.

Les lycéens utilisent leur PC en permanence. De deux à trois heures, les jours scolaires et cinq à six les jours de congé. L'un raconte « une fois, je me suis surpris, j'étais en classe en maths, je sors mon ordinateur portable et je me dis qu'est que je suis en train de faire ? Et je continue ». Une fille l'emporte même aux toilettes ! Dans le domaine des loisirs, notamment du visionnage de films et de séries, l'activité des internautes algériens peut être partagée au sein de la famille ou éventuellement avec des amis, mais elle est le plus souvent solitaire. Zina évoque ses pratiques de lycéenne, «cela m'arrivait de faire des nuits blanches connectées ... je ferme la porte tout le monde pense que je dors ». Cette pratique solitaire d'internet peut prendre des formes limites comme Saida (étudiante à Constantine) qui communique avec sa sœur qui vit dans la même maison qu'elle par internet.

11 Ils ont appris seuls à se servir de leur ordinateur et sont bien souvent les spécialistes de la famille : «Mes parents, dés qu'il y a un problème, c'est Aïda, viens m'aider à faire ci, viens m'aider à faire cela ». Cette expertise leur permet d'utiliser la toile comme bon leur semble ${ }^{10}$.

12 Le contrôle parental semble inexistant, soit par ce que des relations de confiance se sont établies entre les parents et les enfants : «J'avais la connexion à la maison à 13 
ans, on était assez conscient à cette époque là, on savait ce qu'on faisait » (Aicha), soit par ce que les parents n'ont ni les codes, ni les savoir-faire pour surveiller leurs enfants. «Mes parents ils m'ont laissé libre, vu qu'eux ils ne savent pas manipuler les ordinateurs" (Ahmed). On remarque là une curieuse contradiction, les jeunes restent souvent chez eux et pour les filles doivent rester chez elles, mais simultanément, ils mènent leur vie en ligne comme bon leur semble. On retrouve là un constat déjà fait par D. Pasquier à propos des lycéens parisiens, quand la famille n'envoie pas de message clair, les jeunes vont chercher ailleurs (chez les copains ou dans les médias) des repères pour leurs nouvelles pratiques ${ }^{11}$

Cette pratique des outils de communication est aussi largement individuelle. Ce phénomène s'inscrit d'ailleurs dans ce qu'Ulrich Beck considère comme un mouvement bien plus général : « une poussée de l'individualisation d'une intensité sans précédent " depuis le milieu du $20^{\text {ème }}$ siècle ${ }^{12}$. Cette individualisation de la société se manifeste notamment par le refus d'adopter l'identité prescrite par la famille et l'entourage et la volonté de se choisir une identité propre, de la construire, l'envie de pouvoir être véritablement soi-même. Mais comme l'a bien montré Alain Ehrenberg, il ne s'agit pas seulement d'un souhait des individus, mais d'une prescription sociale : «nous sommes désormais sommés de devenir les entrepreneurs de nos propres vies $»^{13}$.

\section{L'intérieur et l'extérieur}

L'individualisation des pratiques de communication qui débouche sur un dispositif de construction de soi a parfois était analysée comme un dispositif de bulle communicationnelle. Déjà Baudelaire au $19^{\text {ème }}$ siècle, célébrait le plaisir de «voir le monde, d'être au centre du monde et rester caché au monde $»^{14}$. Alors que le poète faisait référence au flâneur qui épouse la foule et déambule en emportant en quelque sorte avec lui son espace privé, aujourd'hui, la bulle communicationnelle est installée à l'intérieur de l'espace privé. Les outils communicationnels individualisés contemporains placent l'individu dans une bulle dans laquelle l'extérieur ne pénètre plus $^{15}$. La personne repliée dans sa bulle gère, comme elle le souhaite, ses rapports avec le monde extérieur. Elle choisit les personnes qu'elle veut contacter, les loisirs (musique, vidéo...) auxquels elle peut se consacrer. L'intérieur du domicile constitue ainsi un entre soi protecteur.

Mais grâce aux outils de télécommunications et à internet, cet entre soi peut se connecter au monde extérieur. La vie en ligne permet ainsi de continuer ses contacts interpersonnels sans déplacement et d'accéder à des loisirs de chez soi.

Une sociabilité intense s'organise à travers les réseaux sociaux, mais pas selon n'importe quelle modalité. «Plus on se voit, plus on se téléphone ${ }^{16}$. Ce constat fait il y a une quinzaine d'années sur le téléphone fixe reste vrai aujourd'hui avec la multiplicité des réseaux techniques contemporains. Néanmoins les utilisateurs spécialisent les réseaux. Les jeunes algériens interviewés utilisent conjointement plusieurs dispositifs de sociabilité électronique. Ils les spécialisent dans telle ou telle sphère de la sociabilité : la famille, les amis, les connaissances, les inconnus. Le choix entre ces différents outils peut changer selon les individus. Alors que Zina (19 ans, étudiante en médecine) n'accepte sur Facebook que les personnes qu'elle connaît, Aicha qui fait des études de civilisation étrangère communique avec des Espagnols qu'elle ne connaît pas, dans leur langue, pour apprendre « leurs coutumes, leurs traditions ... on 
discute d'études, de sport... ». Par contre, pour elle, «MSN, c'est personnel ... parce qu'il $\mathrm{y}$ a ta photo, y a beaucoup d'informations te concernant, donc tu n'acceptes pas n'importe qui ». Quant à Skype, c'est aussi un outil de communication le plus intime réservée à la famille qui est à l'étranger, ce sont les seuls interlocuteurs avec qui on peut échanger des images animées. Omar, étudiant à Constantine fait une distinction analogue qu'il exprime plutôt en termes d'aire géographique. MSN, c'est les «gens de Constantine » qu'on peut donc rencontrer en face à face et Facebook les autres, plus éloignés. De même, Ahmed possède deux comptes Facebook, l'un étant réservé à son activité semi-professionnelle de DJ.

17 Les échanges dans le cercle des proches peuvent être aussi organisés dans des communautés en ligne comme celle créée par ces lycéens d'Alger qui ont constitué un groupe Facebook intitulé " première 5 » qui est en quelque sorte leur « cahier de textes commun ". On peut ainsi toujours trouver quelqu'un en ligne qui pourra répondre à vos conseils. Selon un dispositif classique chez les adolescents et les jeunes, la sociabilité en ligne prolonge la sociabilité scolaire ou universitaire. Elle permet non seulement d'obtenir des informations et des renseignements de ses collègues, mais aussi d'exprimer ses émotions : «Facebook c'est aussi un défouloir, des fois quand on est en colère contre quelque chose qui s'est passé en classe, on veut revenir dessus, on publie un statut et les autres commentent» (Soraya). Il y a ainsi un va-et-vient permanent entre les rencontres en face à face et les rencontres en ligne. Les conversations qui ont lieu entre lycéens dans l'enceinte de l'établissement ou à l'extérieur se continuent sur Facebook. Zima, étudiante, issue d'un milieu favorisé fait le même constat à propos de ses sorties avec ses amis : «ce qui est drôle, c'est que quand on est entre amis à la plage, on parle de Facebook et ensuite quand on est sur Facebook, on parle de ce qu'on a fait avant».

Les jeunes chômeurs indiquent également, mais avec plus d'amertume : «Le soir on se retrouve ici [dans la rue] vers le coup de cinq heures, on trouve un carton, on ramène une chaise et on joue aux dominos jusqu'à huit heures du soir et chacun monte chez lui [et on continue sur internet] (...) On fait tout sur Facebook! On drague les filles, on joue... On poste notre humeur et puis voilà, on dit qu'on va bien, on dit qu'on va pas bien ».

Ainsi, contrairement à ce qu'on croit souvent, il n'y a pas de vraie coupure entre le monde réel et le monde en ligne, la sociabilité amicale navigue entre les deux espaces. Il y a néanmoins une spécificité de la communication en ligne, notamment chez les garçons. Comme le dit un lycéen français interrogé par D. Pasquier «il y a des choses qu'on n'oserait pas dire aux gens comme ça en face ... Et, en fait par l'internet, on parle pas, mais on écrit, donc on ose plus $»^{17}$.

20 Mais dès que le monde réel paraît trop clos, le net offre une alternative. C'est ce que constatent deux sociologues françaises à la suite d'une grosse enquête sur « les amitiés virtuelles dans la vie réelle " "il n'y a pas de substitution, puisque ce sont dans l'ensemble des personnes plutôt sociables qui ont des amis virtuels $»^{18}$, néanmoins elles constatent qu'internet peut aussi permettre de s'évader de la vie quotidienne et d'explorer de nouveaux mondes. Le monde en ligne a toujours offert cette possibilité. $\mathrm{Au}$ début de la télématique, Claude Baltz constatait déjà : "la connexion au réseau équivaut à la plongée dans un univers ouvert à toutes les surprises (...) C'est l'aventure à domicile $»^{19}$. 
21 Cette activité d'évasion et d'exploration apparaît notamment dans les domaines où la vie ordinaire est particulièrement frustrante. L'un des premiers domaines où se manifestent ces frustrations est celui des relations avec les filles. « On en rencontre peu en Algérie, parce que les filles en Algérie, c'est pas trop ça ... » dit un jeune chômeur, alors «on parle du genre de filles qu'on aimerait bien avoir ici, chez nous... Malheureusement, on n'en a pas!». Un autre chômeur algérois précise que sur internet, il passe la plupart de son temps à «draguer les filles sur Facebook ». "Sur internet, avec les filles estime Mohamed, on peut aller encore plus loin, on est plus libre qu'en face à face... tu es sûr que la fille te répond ».

22 Un autre garçon (Djamel) observé au centre culturel d'Annaba a plus de 200 amis sur Facebook dont un pourcentage très élevé de filles dont beaucoup à l'étranger, notamment des Américaines. Les échanges sont souvent limités puisque Djamel qui ne connaît pas l'anglais utilise le dictionnaire de Google. Il s'agit d'un jeu de séduction à distance où le plus important est de pouvoir montrer son tableau de chasse à ses amis, car comme l'a bien noté Emmanuel Kessous à la suite d'une observation sur un terrain différent: des sites de rencontre et non Facebook, « la rencontre demande ensuite de renoncer à l'abondance des possibles proposée sur les sites pour prendre acte du caractère limité de l'attention et laisser s'exprimer d'autres registres émotionnels des conduites humaines $»^{20}$. On est face alors à ce qu'Olivier Voirol appelle «les pathologies du soi digital ». «Dans ce cas, les participants de la communication ne sont pas considérés comme des partenaires dont dépend l'extension intersubjective du soi, mais comme de purs instruments de réputation $»^{21}$.

23 Mais pour Djamel, le virtuel n'est donc pas, comme pour les lycéens, une façon de continuer en ligne des contacts dits réels, mais au contraire une façon de rêver, de tenter de construire des relations avec des inconnus, le plus souvent éloignés. Ces relations sont largement fantasmées et irréelles.

24 C'est déjà ce que constatait Sherry Turkle dans Life on the screen, au début d'internet: «vous pouvez être qui vous voulez (...) c'est facile de changer la façon dont les gens vous perçoivent, parce que la seule chose qu'ils connaissent de vous, c'est ce que vous leur montrez. Ils ne regardent pas votre corps et ne font pas de suppositions à partir de là. Ils n'écoutent pas votre accent. La seule chose qu'ils voient c'est vos mots ${ }^{22}$. Ces dispositifs peuvent permettre de se construire un simulacre d'identité, cette seconde $\operatorname{sef}^{23}$ étudiée par Turkle qui a longtemps vu là une forme d'apprentissage social, facilement accessible et sans risque. Aujourd'hui, elle estime plutôt que ces conversations virtuelles ne réussissent pas à pallier une solitude qui demeure. En définitive, l'internaute serait seul avec les autres ${ }^{24}$. Deux visions d'internet s'opposeraient ainsi une perspective d'apprentissage, voire de thérapie et une perspective aliénante.

25 Une analyse plus attentive de l'internet contemporain (web 2.0, réseaux sociaux...) montre que les internautes disposent de multiples dispositifs de présentation de soi qui permettent des modes d'engagement différents dans la "relation virtuelle». Ainsi, Dominique Cardon appréhende la participation aux espaces numériques de deux façons. Tout d'abord un processus de subjectivation expressive par lequel les internautes décrivent leurs goûts et leurs activités, indiquent leurs amis, cela relève du domaine de l'être ou du faire, de l'autre un processus de simulation qui permet aux internautes d'endosser différents rôles, ceux qui correspondent à leur vie "réelle ", ou ceux qui échappent à leur univers quotidien et sont des projections d'eux-mêmes dans 
un autre univers. A partir de ces deux entrées, Cardon dégage plusieurs modèles de participation au monde en ligne, notamment ceux du paravent, du clair-obscur et du phare. Dans le paravent, on est dans un modèle de dissimulation et de masque, dans le clair-obscur, les internautes dévoilent certaines caractéristiques très personnelles d'euxmêmes en profitant de l'opacité du réseau, dans le modèle du phare, les individus peuvent mettre en visibilité certains éléments de leur identité ou de leurs activités ${ }^{25}$. Comme le note olivier Voirol, le monde en ligne est un espace où non seulement l'expression du soi peut être multiple, mais où aussi « le sujet est capable de jouer son identité $»^{26}$.

Les analyses de Cardon et de Voirol montrent bien la complexité des liens virtuels. A cette première cartographie des mondes virtuels, il faut ajouter deux autres dimensions : l'articulation entre les liens en ligne et les liens en face à face, la proximité ou l'éloignement géographique. En effet, certains contacts virtuels ne sont que la prolongation de contacts réels, ils sont réglés par les règles de la communication interpersonnelle avec des proches (famille, amis, connaissances), par contre les autres contacts virtuels qui n'appartiennent pas à la sociabilité familiale et amicale reposent sur d'autres règles : pseudonymat, construction précise d'une présentation de soi, d'un réseau d'amis du net ou du réseau social ...

Même dans le cas de contacts uniquement virtuels, l'espace géographique n'a pas disparu et les internautes interviewés distinguent bien les contacts avec l'intérieur (l'Algérie) et ceux avec l'extérieur (l'étranger), la coupure entre " eux » et "nous » reste toujours perçue. Néanmoins, dans beaucoup de mes entretiens, apparaît un désir d'étranger qui ne concerne pas seulement la sociabilité en ligne, mais aussi les multiples consultations de sites web, de blogs, les personnes suivies sur Twitter... En réfléchissant à ces différentes pratiques, une lycéenne déclare: «j'ai l'impression de vivre plus à l'étranger qu'en Algérie ». Mais cette idée de bulle séparée de l'extérieur n'est pas acceptée par tous. D'autres lycéens la contestent. « Dés qu'on sort dans la rue, on est bien en Algérie» dit une lycéenne, une autre constate néanmoins "mais virtuellement parlant, c'est vrai que je ne suis pas en Algérie, je suis dans un autre monde ». On peut interpréter cette "bulle internet» de plusieurs façons. On peut d'abord considérer qu'il s'agit d'abord d'une bulle sociale : « on vit entre nous, on sort entre nous. On ne côtoie pas l'extérieur (...) On ne voit pas ce qui se passe ailleurs " remarque un élève du lycée. Rappelons qu'on a à faire à des lycéens brillants qui sont élevés dans une culture francophone, largement tournée vers l'extérieur. Ce n'est pas forcément le cas des autres interviewés. La bulle internet est aussi une façon de vivre une autre vie et effectivement le monde numérique peut offrir cette possibilité. On peut penser notamment au slogan de la web TV française, No Life : «Y'a pas que la vraie vie dans la vie ». Quand la "vraie vie » est désespérante, l'autre vie devient encore plus désirable. On peut comme le font les fans s'immergeaient complètement dans un monde de musique, de jeux vidéo ou de mangas. C'est ce que constate ce jeune chômeur d'Alger : «Ici, en Algérie, y a le vide, y a rien ». Ce sentiment est partagé par des jeunes plus favorisés, comme Hosni, élève au lycée Aristote « on est une génération dégoûtée et sans intérêt» et l'une de ses camarades ajoute "une génération triste tout simplement ». Un autre jeune exprimait un sentiment voisin, en déclarant dans une émission de radio : «on s'emmerde collectivement $»^{27}$. Internet est évidemment bien adapté pour rendre plus collectif cet ennui et s'investir dans un monde imaginé. 
Comme le note Francis Jauréguiberry, "Internet serait [alors] un exutoire à une vie trop froide, trop grise ou trop bornée. Au lieu de chercher une solution dans le monde réel, les problèmes de celui-ci seraient fuis par la recomposition virtuelle d'un environnement plus en accord avec les désirs et les attentes de chacun ${ }^{28}$. le voudrait le héros du film de Tariq Teguia « Rome, plutôt que vous ", mais à défaut on peut voyager dans le cyberespace. Pour une petite minorité, certains élèves du lycée Aristote, il faut vraiment aller à l'étranger. « Mes parents m'ont dit, indique Malika, ma fille tu as ton bac et te sauves, parce qu'ici, y a pas d'avenir ». Ce n'est pas une fuite précise l'un de ses camarades, c'est "parce qu'on veut réussir notre vie tout simplement! ».

Que l'on puisse ou non quitter l'Algérie, Internet est absolument central pour ces étudiants, quand l'enquêteur évoque la possibilité d'une disparition du réseau, une jeune fille sage de 18 ans (Aicha) lui répond "on se suiciderait». Devant mon étonnement, elle indique qu'elle plaisante, mais un peu plus tard, elle déclare "vivre sans, c'est impossible! ». Des expressions analogues reviendront chez un bon tiers des interviewés. "Je serais étouffée " déclare Djamina, une interne en médecine de Constantine. Elle ajoute «Si je n'ai pas internet, je peux rien faire, je ne peux pas joindre mon mari [qui travaille en France], ni le monde, je suis isolée ». L'attachement à internet apparaît donc d'autant plus grand que le sentiment d'enfermement dans le domicile ou dans un espace géographique désespérément vide qui n'offre aucune opportunité est vécu fortement. Ce n'est évidemment pas un hasard, si cet attachement est exprimé le plus fortement par les filles et par les chômeurs.

\section{Le numérique, comme ressources nouvelles}

31 Internet n'est pas seulement un espace virtuel qui permet de prolonger en ligne les contacts de la vie réelle ou de se projeter dans un autre monde, c'est aussi un ensemble de ressources qui permettent de faire ses études plus efficacement, d'accéder à des nouveaux programmes musicaux, vidéo ou tout simplement de pouvoir observer la rumeur du monde.

\section{Etudier avec l'appui d'internet}

Internet est largement utilisé par tous les interviewés pour leurs études. Ceux-ci sont à l'affût de ressources scolaires ou universitaires en ligne. Ainsi, Bachir, étudiant en Master de lettres à Alger "ne peux pas travailler sans internet, c'est impossible », il regarde notamment sur les chaînes françaises ou dans les archives de l'Ina des conférences ou des débats avec des grands intellectuels vivants ou décédés, comme Deleuze, Badiou, Chomsky, Bourdieu, ...

Pour Omar étudiant en master de marketing à Constantine, internet «est un instrument de travail en premier lieu ». Ses enseignants lui envoient leur cours de cette façon, et ses collègues qui habitent en résidence universitaire et ne disposent pas du Wifi doivent aller dans des cybercafés pour accéder à leurs cours. Il poursuit ses études en ligne en suivant une formation par internet en France. De même Naïma, étudiante en urbanisme à Constantine, s'exerce à la préparation de plan d'urbanisme à l'aide 
d'informations trouvées en ligne. Elle a appris seule à se servir des logiciels de dessin ou de photo, comme AutoCad ou Photoshop, en demandant conseil par mail ou par discussion instantanée sur Facebook à des amis. Djamina, quant à elle termine ses études de médecine à Constantine, elle télécharge le Vidal. Kamel qui est interne en médecine dans la même ville va chercher sur internet les livres qu'il ne trouve pas dans sa bibliothèque. Une enquête récente réalisée en France (à Rouen) et en Algérie (à Batna) sur l'utilisation du web par les étudiants en médecine confirme cette déclaration. Elle montre qu'internet est un outil central pour les étudiants de Batna qui à $45 \%$ estiment qu'internet est plus utile que les livres pour préparer leurs examens. Leurs collègues de Rouen accordent moins d'importance à internet puisqu'ils ne sont que $10 \%$ à faire la même déclaration ${ }^{29}$.

Pour Amine, étudiant en Relations humaines à Alger, le web est un instrument central pour préparer ses exposés, avec l'aide de Google et de Wikipedia. C'est également là qu'il peut se tenir au courant de l'évolution des méthodes managériales. Bachir échange avec ses professeurs et les autres étudiants. Internet est donc utilisé de façon multiple dans le travail scolaire et universitaire. Il permet d'accéder à des ressources en ligne, pour compléter des bibliothèques dont les collections sont limitées et des formations insuffisantes. Il constitue aussi un vaste terrain d'enquête où les étudiants doivent chercher la matière de leur exposé ou de leur mémoire. Le net permet enfin d'organiser la coopération avec les autres élèves ou les autres étudiants. Dans des situations où les ressources sont insuffisantes, internet apparaît vraiment comme un outil de démocratisation des connaissances.

\section{Les loisirs à l'intersection de plusieurs univers culturels}

Mais internet est aussi un outil qui permet d'accéder à d'autres univers de loisirs et notamment à des produits culturels nombreux et diversifiés.

Le visionnement des films et des séries, le plus souvent américains constitue une activité particulièrement appréciée. Elle peut occuper des journées entières et est souvent organisée de façon solitaire. C'est un comportement de lycéenne, comme celui de Malika qui a profité de l'absence de ses parents pour regarder huit films de suite, mais aussi d'étudiante en fin d'études, comme Naïma qui regarde des séries en continu. "J'arrête juste pour manger et je reviens tout de suite ». Kamel, interne en médecine, peut également consacrer un week end à voir une saison entière d'une série.

Films et séries peuvent être regardés sur le poste de télévision, en fonction des opportunités de la programmation, mais les titres les plus appréciés sont regardés quand on en a envie, souvent en fonction des conseils des amis. Le visionnement se fait sur internet en streaming, ou à la suite d'un téléchargement. On s'échange aussi les films sur des clés USB ou des DVD qui sont particulièrement peu chers, parce qu'ils sont le plus souvent piratés. Hosni, lycéen télécharge ainsi des films en permanence, il en a ainsi téléchargé 150 l'année précédente. Comme la pratique des mondes virtuels évoquée plus haut, l'immersion dans les séries télévisuelles n'est pas cette fuite devant la réalité dénoncée par certains. Comme Ien Ang l'a montré à propos de Dallas, la fiction est moins séparée de la réalité qu'on ne le croit. Elle parle de "réalisme émotionnel ", c'est-à-dire que si l'univers de Dallas est bien différent de celui des téléspectateurs, les émotions éprouvées à l'écran ressemblent à celles que le spectateur ressent. « La 'fuite' dans un univers de fiction n'est pas tant une négation de la réalité 
qu'un jeu avec elle. Un jeu qui permet à la personne de mettre en question les limites de la fiction et du réel pour les rendre fluides. Et au cours de ce jeu, une participation imaginaire à l'univers de fiction est vécue comme un plaisir $»^{30}$.

En matière musicale, les modes de réception sont également individualisés. On écoute intensément ce qu'on veut, quand on veut, grâce à internet. Djamina écoute aussi bien de la musique constantinoise que de la musique française (Enrico Macias, Céline Dion). Kamel écoute plutôt du pop, du rock ou du rap. L'accès à la musique se fait à travers You Tube, pour Omar. Naima écoutait Deezer, jusqu'au jour où l'accès a été bloqué. Si l'écoute est individuelle, les interviewés rentrent néanmoins dans un jeu de recommandations qui leur permet de signaler les morceaux qu'ils aiment. "J'ai l'habitude de publier, une ou plusieurs fois par jour des liens de You Tube sur Facebook avec des morceaux qui me plaisent ou des morceaux que je découvre, de façon à les publier et à les partager ", déclare Malika.

Le sport international est également accessible par internet. Les jeunes chômeurs d'Alger qui sont amateurs de foot sont des supporters des grandes équipes européennes : Arsenal, le FC Barcelone, le Real Madrid ... Comme Hosni, ils regardent en ligne les matchs qui ne passent pas à la télévision algérienne. Ils préfèrent largement ces matchs à ceux des équipes algériennes qui d'après eux n'ont pas la même qualité technique. De même, Omar qui est un passionné de sport de combat, activité qu'il pratique, va voir sur You Tube les grandes compétitions internationales.

\section{La rumeur du monde}

Internet offre enfin l'avantage de pouvoir observer le monde extérieur. On peut tout d'abord assister à la vie des autres. Malika, dés qu'elle rencontre des amis, prend des photos qu'elle met sur Facebook, "c'est comme si c'était pour immortaliser le moment ». Soraya est toujours à l'affût de ce que font les autres: "On a des amis qui publient des vidéos aussi, ce qui nous permet de découvrir beaucoup de choses. Personnellement, moi, dès que quelqu'un publie quelque chose, je vais voir de quoi il s'agit ».

Pour Zina, 19 ans qui passe parfois des "nuits blanches, connectées ", la première activité est d'observer la vie de ses connaissances : « si on sait pas qui a publié, qui a dit quoi, on s'ennuie. Enfin, surtout quand on a rien à faire et comme en Algérie, y a pas grand-chose à faire... » Cette observation de la vie des autres s'étend à l'étranger : « cela nous permet de voir un peu ce qu'ils vivent » surtout s'ils publient des photos. Hosni, grâce à Facebook suit des personnes qui font des vidéos, « cela me permet de le savoir immédiatement, pour pouvoir aller les regarder ». Cette pratique des jeunes algériens n'est pas sans ressemblance avec celle des pionniers d'un des premiers sites communautaires du net, The Well. Sur l'un des forums, deux protagonistes évoquaient quotidiennement leurs amours tumultueuses. Les autres internautes voyaient là une nouvelle série de télévision qu'ils commentaient abondamment. Ils étaient les familiers et les complices de ces deux personnes qui étaient devenus en quelque sorte des stars du net ${ }^{31}$. Aujourd'hui, cette starisation de la présentation de soi n'est plus une exception, mais plutôt la norme sur les blogs ou sur Facebook, ce qui fait que suivre en ligne des individus ordinaires ou des stars n'est plus très différent.

41 Pour certains interviewés, ce spectacle du monde s'arrête à la vie des autres. «J'ai beau essayé, dit Zina, j'arrive pas à m'intéresser à tout ce qui est information et tout... ». Par 
contre Amine suit avec passion le spectacle de la vie politique à l'étranger et tout particulièrement en France. "C'est un spectacle de démocratie ... Personne ne voyait les élections algériennes, mais on voyait les élections de France, qui va être Président?». Ce spectacle peut être même réellement divertissant. Amine note à propos de Nicolas Sarkozy : «il me fait rire des fois, il gesticule trop ... j'aime trop le regarder ». Bachir, qui avait déjà suivi la campagne électorale française de «très, très prêt » a aussi des opinions précises sur la vie politique française : «si j'étais français, j'aurais voté Mélenchon au premier tour et je m'abstiendrais au deuxième ».

42 Les élèves du lycée Aristote lisent plutôt la presse en ligne, ils reçoivent le flux RSS d'El Watan, du Monde ou du Courrier international. Cet attrait pour le numérique semble largement partagé par le public du sud de la Méditerranée. A l'issue d'une enquête menée dans les pays du pourtour méditerranéen sur les modes de recherche de l'information sur le Printemps arabe, trois chercheurs constatent que les dans les pays du sud on utilise plutôt les nouveaux médias, alors que dans ceux du nord on privilégie les médias classiques ${ }^{32}$.

Pour Malika « quand on va sur internet, on suit plus le fil des actualités internationales, parce que l'actualité algérienne, on la vit, on la subit quelque part ». La rumeur du monde est toujours plus attirante que la rumeur locale.

\section{Mener une double vie : découvrir et cultiver sa passion}

Dans une grande enquête statistique menée en France en 2003 intitulée Histoire de vie, on demandait aux enquêtés in fine les trois éléments fondateurs de leur identité. Deux grands domaines ressortaient: la sociabilité (la famille et les amis) et le "faire »: le métier et les passions ou hobbies. Chez les actifs, le travail arrive très nettement en tête (59\%) avant les passions (24\%). Par contre chez les étudiants, le travail (d'apprentissage dans ce cas) est presque aussi choisi que les passions (46\% contre $38 \%$ ). Les passions sont donc bien un élément important de l'identité des jeunes.

Pour bien comprendre les raisons de l'attachement au travail universitaire et aux passions, on peut avec les théoriciens du don, comme Alain Caillé distinguer deux motifs de l'action humaine : l'« intérêt à », qui relève de l'instrumentalité, de ce que l'action rapporte à l'individu en termes d'usage, de salaire ou de notoriété, et l'« intérêt pour ", où l'action est à elle-même sa propre fin (on est dans l'ordre du plaisir pour l'action ou pour le destinataire de cette action) ${ }^{33}$. Dans cette théorie du don, celui-ci n'est pas uniquement altruiste; il n'est pas synonyme de désintéressement, mais il articule "intérêt pour » et "intérêt à ». Si le don s'oppose par définition au travail salarié, les deux activités associent malgré tout, selon des proportions différentes, « intérêt pour " et « intérêt à », plaisir et contrainte.

Les passions reflètent la volonté de l'individu de construire son identité, de favoriser son épanouissement personnel, de développer des activités qui lui soient propres, d'agir pour son plaisir. C'est le règne de l'« intérêt pour ». L'individu peut trouver là des satisfactions que ne lui apportent pas toujours ses études. Sa quête identitaire l'amène à exprimer ses talents, à mettre en scène sa singularité face aux autres. L'élaboration de son identité numérique lui permet à la fois de se distinguer, d'être reconnu et de construire des liens. Mais l'amateur n'est pas pour autant indifférent aux motivations de l'« intérêt à » : s'il est rare qu'il obtienne une rémunération financière, il peut retirer de son activité des gratifications symboliques (par exemple la fierté ou la notoriété). 
Grâce à internet, l'amateur peut facilement accéder aux ressources cognitives dont il a besoin pour pratiquer ses activités. L'internaute dispose, comme n'importe quel travailleur intellectuel, d'un commentaire diversifié sur son activité, aussitôt qu'elle est mise en ligne. Il reçoit des encouragements, des critiques, des corrections, surtout si son activité s'insère dans un travail collectif. Si sa production figure sur une plateforme de blogs ou un site de partage, il dispose par surcroît de données sur les "visiteurs " qui se sont intéressés à sa production. En bref, sa construction identitaire se fait en réseau.

Comme l'avait déjà remarqué D. Pasquier, en France, les passions personnelles apparaissent essentiellement chez les jeunes garçons ${ }^{34}$. La plupart des jeunes algériens que nous avons interviewés affichent une passion personnelle. Si celle-ci se nourrit d'internet qui apporte des ressources nouvelles, des exemples à suivre, des conseils, néanmoins les passions présentées par ces garçons se pratiquent dans le monde « réel », à l'extérieur du domicile. On comprend alors que les filles qui sortent peu n'indiquent pas de passions au cours des entretiens.

J'ai déjà évoqué la pratique du sport de deux interviewés, pour eux Internet est d'une façon de passer de la position du sportif amateur à celle du fan qui vient regarder ses vedettes. Pour Adel qui fait des études d'ingénieur, la danse hip-hop est plus qu'une passion, c'est une deuxième vie, la troupe semi-professionnelle à laquelle il participe a réalisé de nombreuses tournées tant en Algérie, qu'à l'étranger. Elle dispose bien sûr d'un site web, avec des vidéos de ses spectacles et d'une page Facebook. Sur cette page qui est principalement fréquentée par des danseurs, on parle notamment des nouvelles techniques de danse.

49 Ahmed qui est en terminal a aussi une autre vie, celle de DJ. Il a découvert la house music sur internet, («sinon, j'aurais jamais découvert ces musiques ... Franchement, sans internet, j'aurais pas fait carrière ») et petit à petit il a développé ses compétences de DJ. Sa page "professionnelle» de Facebook lui permet de se faire connaître et de trouver des engagements tous les soirs d'été. Il y publie ses "apparitions en live». Hosni, lui est un passionné de tour de magie. Il a fait le tour des accessoires grand public qu'on peut trouver à Alger. Pour développer sa passion, il va sur internet, car ce que note D. Pasquier pour les lycéens parisiens est encore plus vrai à Alger «plus la passion est peu commune, plus le rôle du Net est grand, [car] il est difficile de recruter d'autres passionnés dans l'entourage direct $»^{35}$

50 Pour bien comprendre, ce rôle d'internet, il est intéressant de le comparer à celui joué autrefois par le minitel. Josiane Jouët ${ }^{36}$ rappelle que les amateurs avaient essayé d'investir les messageries minitel pour échanger sur leurs pratiques. Ces tentatives ont largement échoué, car ces messageries ne permettaient pas de partager des documents. Internet au contraire autorise ce partage. C'est à travers lui que s'échangent et se démocratisent les compétences.

51 Amine lui est passionné de photo et de vidéo, il filme les aspects insolites d'Alger et les met en ligne. Il est aussi l'animateur d'une association d'étudiants qui vient en aide aux enfants défavorisés. Il s'est inspiré d'une association dont il a vu la présentation à la télévision, sur une chaîne française. De même Kamel est membre d'un «petit mouvement écolo algérien » qui fonctionne principalement en ligne à travers une page Facebook. Les membres de Constantine se réunissent parfois dans un café.

52 Soraya, élève au lycée Aristote. est la seule fille que j’ai interviewé qui affiche une passion qu'elle exerce uniquement en ligne. Elle a ouvert un blog BD. «Je fais des 
petites BD ... ça me permet en même temps de m'exprimer, en même temps de m'améliorer en dessin. Et des gens plus expérimentés que moi ou des professionnels ou des amateurs, ils me laissent des commentaires en me disant ce qu'il faut améliorer, par exemple, ça, c'est tordu, ça, t'aurais pu mettre une autre couleur... » Elle soigne la notoriété de son blog: "En m'inscrivant sur des annuaires, en le donnant à mon entourage et puis, en laissant des commentaires sur d'autres blogs aussi avec le lien de mon blog, comme ça, les gens, ils ont le réflexe de cliquer et de venir voir. Aujourd'hui, j'ai quand même assez de visites, une trentaine, quarantaine par jour et des commentaires ». Elle surveille ses statistiques et a pu noter qu'un tiers de ses visiteurs était algérien et les autres étrangers. Finalement, ces passions n'isolent pas l'individu, car, comme le note François de Singly, l'investissement dans une passion produit une compétence qui «sert de support aux échanges avec les personnes qui partagent le même amour de la pratique $»^{37}$.

Pour ces passionnés, internet est aussi bien une ressource qui permet à Ahmed de découvrir une musique qu'il ignorait ou à Hosni d'augmenter ses compétences de prestidigitateur, qu'un dispositif qui permet de rendre visible son activité (le modèle du phare de Cardon) et d'accroître sa réputation, comme Kamel avec la danse hip-hop et Soraya avec son blog BD. Comme le note Voirol, internet offre simultanément de nouvelles formes "d'exploration de soi » (découverte d'autres manières d'être et d'agir » et «d'exposition de soi » (se mettre en scène et tester ses pratiques face aux autres) ${ }^{38}$.

\section{Conclusion}

Ces jeunes algériens, par bien des aspects, ont une pratique d'internet qui ressemble beaucoup à celles de jeunes étudiants européens, comme si le réseau des réseaux homogénéisait les pratiques de la jeunesse. Ces jeunes ont néanmoins une spécificité, ils ont accès à moins de ressources, tant en matière d'études, que de loisirs et de sociabilité. Leur intégration dans internet est d'autant plus grande que la vie en ligne permet de suppléer aux ressources auxquels ils n'ont pas accès. Si la vie de ces jeunes apparaît bien souvent vide, bloqué, internet peut aussi être un formidable outil de découverte, un espace qui permettrait de construire une autre vie.

On pourrait alors considérer qu'internet ne serait qu'une illusion, un pauvre substitut à des relations amoureuses bloquées, à un espace public défaillant, à l'absence de loisirs diversifiés. Cela reviendrait à considérer qu'internet est en dehors de la "vraie vie ", qu'il permet de s'immerger ailleurs. En réalité, il faut plutôt considérer que le réseau des réseaux est un élément important de la vie de ces jeunes, qu'il est intégré aux différentes facettes de leur vie sociale dans le cadre d'un environnement qui tend à les replier sur leur domicile et leur pays.

Internet présente des configurations différentes selon les groupes rencontrés. Pour les jeunes chômeurs algérois qui ne font plus d'études, n'ont pas de perspectives professionnelles, rencontrent difficilement des filles, internet permet de continuer la vie en bande, quand on n'est plus ensemble dans la rue, d'accéder à sa passion d'amateurs des grands clubs de foot, d'échafauder des relations masquées, mais plus apaisées avec l'autre sexe. A l'inverse, le réseau des réseaux permet aussi de rêver à l'impossible vie à l'étranger. 

aide précieuse pour leurs activités scolaires ou universitaires, leur permet de développer une sociabilité amicale intense depuis chez eux, leur offre des loisirs diversifiés à domicile, et pour une bonne partie d'entre eux les prépare à vivre dans le monde international où ils voudraient vivre. Pour tous, internet est un élément essentiel de la construction de soi, de cet "individualisme expressif " $^{39}$ qui se fabrique dans le «laboratoire social identitaire $»^{40}$ qu'est internet. C'est évidemment pour les filles que le réseau des réseaux est le plus essentiel, il leur permet de gérer le repli sur l'espace privé qui leur est imposé.

En définitive, pour tous nos interviewés, la vie en ligne permet d'être à la fois ici et ailleurs. Le réglage entre ces deux espaces est différent selon les uns et les autres. On peut être essentiellement ici et aller chercher à l'extérieur les ressources dont on a besoin. On peut, à l'inverse, vivre avant tout ailleurs et trouver aussi dans internet les connexions avant le réseau amical et familial qui vous ancrent ici. On peut aussi rester enfermé chez soi et observer avant tout la rumeur du monde. Finalement, la plupart réussissent à bien jongler entre l'ici et l'ailleurs, trouvant ailleurs les ressources qu'ils n'ont pas ici, gérant l'espace public depuis leur espace privé individuel, essayant de surmonter les difficultés ou les blocages de leur société, notamment la rencontre de l'autre sexe, la participation à la vie citoyenne ou politique, le départ vers d'autres pays. Quand l'immersion dans internet est intense et permet de se plonger dans un ailleurs largement imaginaire, faut-il considérer que c'est une fuite ou la façon pour les individus de gérer les contraintes de leur environnement, car vivre en ligne, c'est avant tout vivre.

\section{BIBLIOGRAPHIE}

Bibliographie

Alain Caillé, Don, intérêt et désintéressement. Bourdieu, Mauss, Platon et quelques autres, Paris, La Découverte, MAUSS, 1994.

Alain Ehrenberg, Le culte de la performance, Paris, Calmann-Lévy, 1991.

Baudelaire C., « Le peintre de la vie moderne » in Euvres complètes, Paris, La Pléiade, Gallimard, 1961, pp. 1152-1192.

Claude Baltz., « MSG Gretel: Images de personne(s) » Réseaux 1984 n 6, pp.3-19.

Dominique Cardon, « Le design de la visibilité. Un essai de cartographie du web 2.0 » Réseaux, $\mathrm{n}^{\circ}$ 152, 2008, pp.7-17.

Dominique Pasquier, Cultures lycéennes. La tyrannie de la majorité. Paris, Autrement, 2005.

Emmanuel Kessous, «L'amour en projet : Internet et les conventions de la rencontre amoureuse » Réseaux, n 166, 2011, pp.191-223.

Communication, technologies et développement, 2 | 2015 
Emmanuelle Crenner, « La parenté : un réseau de sociabilité actif mais concentré » INSEE Première Juillet, $\mathrm{n}^{\circ}$ 600, 1998, pp.

Francis Jauréguiberry, Les branchés du portable, Paris, PUF, 2003.

François Singly (de), Les uns avec les autres : quand l'individualisme crée du lien, Hachette, Paris, 2005.

Ien Ang, Watching Dallas, Methuen, Londres 1985, cité par Kim Christian Schröder, «Vers une convergence de traditions antagonistes ? Le cas de la recherche sur le public ", Réseaux, 1985, vol. $9, \mathrm{n}^{\circ} 44-45, \mathrm{pp} .315-338$.

Joël Ladner et alii « Utilisation de l'Internet chez les étudiants en médecine à Batna, Rouen et Sousse » Santé Publique, 2010, pp.637-646.

Josiane Jouët, « Des usages de l'informatique aux Internet Studies » in J. Denouël et F. Granjon op. cit. 2011.

Josiane Jouët, « Des usages de la télématique aux Internet studies » in J. Denouël et F. Granjon Communiquer à l'ère numérique Paris, Presses des Mines, 2011.

Laurence Allard, «L'impossible politique des communautés à l'âge de l'expressivisme digital », Sens Public, 2008, n 7-8, pp.105-126.

Laurence Allard, Frédérick Vandenberghe, « Express yourself ! Les pages perso » Réseaux 2003, n 117, pp. 191-219.

Olivier Donnat, Les pratiques culturelles des français à l'ère numérique. Enquête 2008, Paris, La Découverte, 2008.

Olivier Martin, «L'internet des 10-20 ans. Une ressource pour une communication autonome » Réseaux, n¹23, 2004, pp.25-58.

Olivier Martin, François Singly (de), «L'évasion amicale. L'usage du téléphone familial par les adolescents » Réseaux, n 103, 2000, pp. 91-118.

Olivier Voirol, «L'intersubjectivation technique : de l'usage à l'adresse. Pour une théorie critique de la culture numérique » in J. Denouël et F. Granjon op. cit., 2011.

Patrick Flichy, (), Une histoire de la communication moderne, Paris, La Découverte, 2004.

Patrick Flichy, L'Imaginaire d'internet, Paris, La Découverte, 2001.

Patrick Flichy, Le sacre de l'amateur. Sociologie des passions ordinaires à l'ère numérique, Paris le Seuil, 2010.

Piermarco Aroldi, Fausto Colombo, «Questioning ‘digital global generations'. A critical approach » Northern Lights 2013, Vol. 11, pp.175-190.

Sherry Turkle, Alone Together: Why we Expect More from Technology and Less from Each Other, New York, Basic Books, 2011.

Sherry Turkle, Life on the Screen. Identity in the Age of the Internet, New York,Touchstone, 1997.

Sherry Turkle, Second Self: Computers and the Human Spirit, Cambridge (Ma), MIT Press, 2005.

Sonia Livingstone, «Les jeunes et les nouveaux média. Sur les leçons à tirer de la télévision pour le PC » Réseaux, n 92-93, 1999, pp.103-132.

Ulrick Beck, La société du risque : sur la voie d'une autre modernité, Paris, Flammarion, 2008.

Virginie Lethiais, Karine Roudaut, «Les amitiés virtuelles dans la vie réelle. Profils, motifs et modalités de construction », Réseaux n 164, 2010, pp.13-49. 
Younes Boughzala, Inès Bouzid, Jean Moscarola, « Le rôle des réseaux sociaux et des TIC dans les révolutions arabes : les résultats d'une enquête » IREGE, Université de Savoie, 2012.

Zbigniew Smoreda, Présentation du dossier « Cycles de vie et sociabilité » Réseaux n 115, 2002, pp.9-13.

\section{NOTES}

1. Sur cette question, voir Pier-Marco Aroldi et Fausto Colombo "Questioning 'digital global generations'. A critical approach » Northern Lights 2013 Vol. 11 p. 175-190

2. J'ai réalisé, en octobre 2011, quinze entretiens individuels : 7 à Alger, 6 à Constantine, 2 à Annaba. Par ailleurs, j'ai fait deux entretiens de groupe avec une vingtaine de lycéens d'un établissement d'excellence, l'autre dans le centre d'Alger, avec un groupe d'une petite dizaine de jeunes chômeurs. Les entretiens ont été réalisés en français, avec des jeunes qui maniaient bien cette langue.

3. Dominique Pasquier Cultures lycéennes. La tyrannie de la majorité. Paris, Autrement, 2005

4. Pour l'essentiel, les jeunes interviewés disposent presque tous d'un ordinateur personnel connecté à internet depuis déjà plusieurs années. Certains ont commencé à aller sur le net au début de l'adolescence, dans des cybercafés au démarrage, puis sur l'ordinateur familial, et enfin sur leur PC portable. L'accès aux logiciels quasiment tous piratés ne semble pas poser de problème ni économique, ni d'apprentissage. La pratique d'internet est intense, Facebook est très largement utilisé.

5. Voir Olivier Donnat Les pratiques culturelles des français à l'ère numérique. Enquête 2008, La découverte, 2008, p. 179-180

6. Les prénoms ont été changés

7. Olivier Martin et François de Singly «L'évasion amicale. L'usage du téléphone familial par les adolescents » Réseaux $2000 \mathrm{n}^{\circ} 103$

8. Sur l'analyse de ces évolutions, voir Patrice Flichy Une histoire de la communication moderne. La découverte, Paris, 2004, Sonia Livingstone «Les jeunes et les nouveaux média. Sur les leçons à tirer de la télévision pour le PC » Réseaux 1999, n 92-93

9. En 2010, 77\% des internautes algériens se connectaient depuis chez eux, $12 \%$ du bureau et $7 \%$ depuis un cybercafé ou un lieu public (Source Etude Webdialn@). D'autres sources (El Watan 17/05/2010) donne une place beaucoup plus importante au cybercafé.

10. Là encore la situation n'est pas propre à l'Algérie, pour la situation française, voir D. Pasquier op. cit. p. 36 et Olivier Martin «L'internet des 10-20 ans. Une ressource pour une communication autonome » Réseaux $2004 \mathrm{n}^{\circ} 123$

11. D. Pasquier op. cit. p. 165

12. Ulrich Beck La société du risque : sur le voie d'une autre modernité, Flammarion, Paris, 2008, p. 158

13. Alain Ehrenberg, Le culte de la performance, Paris, Calmann-Lévy 1991, p.16.

14. Charles Baudelaire « Le peintre de la vie moderne » in Euvres complètes, La Pléiade, Gallimard, Paris, 1961, p. 1160

15. Voir P. Flichy 1994 op. cit. Francis Jauréguiberry Les branchés du portable, PUF, Paris, 2003 Voir P. Flichy 1994 op. cit.

16. Emmanuelle Crenner «La parenté : un réseau de sociabilité actif mais concentré » INSEE Première Juillet 1998, n 600. Voir également Zbigniew Smoreda Présentation du dossier «Cycles de vie et sociabilité » Réseaux 2002 n 115

17. D. Pasquier op. cit. p.152 
18. Virginie Lethiais et Karine Roudaut «Les amitiés virtuelles dans la vie réelle. Profils, motifs et modalités de construction » Réseaux 2010, n 164, p. 44

19. Claude Baltz "MSG Gretel: Images de personne(s)" Réseaux 1984 nº 6, p. 11

20. Emmanuel Kessous «L'amour en projet: Internet et les conventions de la rencontre amoureuse » Réseaux 2011, n 166 p. 197

21. Olivier Voirol «L'intersubjectivation technique: de l'usage à l'adresse. Pour une théorie critique de la culture numérique » in J. Denouël et F. Granjon op. cit. p. 153. Pour une analyse précise d'un cas de pathologie du soi digital voir le travail de Ouahiba Aboubeker qui a observé des jeunes d'une ville moyenne algérienne : Sidi Bel Abbès.

22. Sherry Turkle Life on the Screen. Identity in the Age of the Internet Touchstone, New York, 1997, p. 184

23. Sherry Turkle Second Self: Computers and the Human Spirit, MIT Press, Cambridge (Ma), 2005

24. Sherry Turkle Alone Together: Why we Expect More from Technology and Less from Each Other, Basic Books, New York, 2011

25. Dominique Cardon « Le design de la visibilité. Un essai de cartographie du web 2.0 » Réseaux $2008, \mathrm{n}^{\circ} 152$, p. 98à 104. Une réflexion analogue avait déjà été engagée par Josiane Jouët sur le dispositif qui a été développé en France dans les années 1980, le minitel. Voir « Des usages de la télématique aux Internet studies » in Julie Denouël et Fabien Granjon Communiquer à l'ère numérique Presses des Mines, Paris, 2011

26. O. Voirol op. cit. p. 139

27. Cité sur France Culture, 16 mars 2012 Emission « Avoir 20 ans en Algérie »

28. Francis Jauréguiberry « Le moi, le soi et Internet » Sociologie et Société, 2000 n², p. 143

29. Joël Ladner et alii « Utilisation de l'Internet chez les étudiants en médecine à Batna, Rouen et Sousse » Santé Publique, 2010/6 p. 641

30. Ien Ang Watching Dallas, Methuen, Londres 1985, cité par Kim Christian Schröder, «Vers une convergence de traditions antagonistes ? Le cas de la recherche sur le public ", Réseaux, vol. 9, $\mathrm{n}^{\circ}$ 44-45, p. 333

31. Patrice Flichy L'imaginaire d'internet, Paris, la Découverte, 2001. J'ai fait également des observations voisines sur l'usage de Facebook chez des étudiantes françaises vivant en grande banlieue parisienne.

32. Younès Boughzala, Inès Bouzid, Jean Moscarola « Le rôle des réseaux sociaux et des TIC dans les révolutions arabes : les résultats d'une enquête » IREGE, Université de Savoie, 2012

33. Alain Caillé, 1994. Don, intérêt et désintéressement. Bourdieu, Mauss, Platon et quelques autres, Paris, La Découverte, MAUSS.

34. D. Pasquier op. cit. p. 97 et suivantes. .

35. D. Pasquier op. cit. p. 103

36. Josiane Jouët « Des usages de l'informatique aux Internet Studies » in Julie Denouël et Fabien Granjon op. cit. p. 69

37. François de Singly Les uns avec les autres : quand l'individualisme crée du lien, Hachette, Paris, 2005, p. 124

38. O. Voirol op. cit. p. 131-132

39. Laurence Allard et Frédéric Vandenberghe «Express yourself! Les pages perso " Réseaux $2003, \mathrm{n}^{\circ} 117$

40. Laurence Allard, « l'impossible politique des communautés à l'âge de l'expressivisme digital », Sens Public, $\mathrm{n}^{\circ}$ 7-8, 2008, p. 118 


\section{RÉSUMÉS}

L'enquête qualitative présentée ici porte sur l'usage d'internet par les lycéens et les étudiants francophones algériens. Ils trouvent là un dispositif qui leur fournit une aide précieuse pour leurs activités scolaires et universitaires, leur permet de développer une sociabilité amicale intense depuis chez eux, leur offre des loisirs diversifiés à domicile. C'est évidemment pour les filles que le réseau des réseaux est le plus essentiel, il leur permet de gérer le repli sur l'espace privé qui leur est imposé. La plupart de ces jeunes, réussissent à bien jongler entre l'ici et l'ailleurs, trouvant ailleurs les ressources qu'ils n'ont pas ici, gérant l'espace public depuis leur espace privé individuel, essayant de surmonter les difficultés ou les blocages de leur société, notamment la rencontre de l'autre sexe, la participation à la vie citoyenne ou politique, le départ vers d'autres pays.

A qualitative survey carried out on Algeria shows that French-speaking students find in the internet a great help for their studies, a tool for strong sociability and many leisure facilities. For the girls who are often housebound, the internet is a very important way to be connected to the world. Most young people move easily from here to elsewhere. They find elsewhere intellectual resources, they don't have here, and they organize public space from private one. They try to overcome constraints in Algerian society, especially to interact with the other sex, to find spaces for political and citizen participation, to exchange with foreigners.

\section{INDEX}

Keywords : Algeria, Internet, Students, Private, Public

Mots-clés : Algérie, Internet, étudiants, privé, public

\section{AUTEUR \\ PATRICE FLICHY}

Professeur de Sociologie à l'Université Paris Est 